Review Paper:

\title{
An Overview of Artificial Intelligence Applications and Psychology
}

\author{
Mohammad Tahan ${ }^{1 *}$ (D) \\ 1. Young Researchers and Elite Club, Birjand Branch, Islamic Azad University, Birjand, Iran.
}

\begin{tabular}{|c|c|}
\hline $\begin{array}{l}\text { Use your device to scan } \\
\text { and read the article online }\end{array}$ & \\
\hline 口pher & Citation Tahan M. An Overview of Artificial Intelligence Applications and Psychology. Avicenna Journal of Neuropsychophysiol- \\
\hline 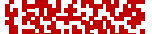 & \\
\hline 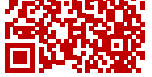 & doil'http://dx.doi.org/10.32598/ajnpp.5.1.3 \\
\hline
\end{tabular}

\section{(c) (4) (8)}

Article info:

Received: 10 May 2018

Accepted: 25 Oct 2018

Available Online: 01 Feb 2018

Keywords:

Artificial Intelligence, Applications, Psychology

\begin{abstract}
Psychotherapy endows a sense of control in patients gripped with emotional conflicts; it allows them to handle their reflexes and regain composure through conscious and behavioral alterations. These transformational changes can be brought about by improving their listening skills, observational capacities, creating awareness, and making them more attentive and intervening. Conventional psychotherapy calls for one-to-one sessions during treatment. Considering the increased access to Information Technology (IT) in our daily living, the idea of replacement of human interaction by IT tools appeared. The specialized tools and techniques are employed through the course of therapy, which not only alters but also accentuates their cognitive and practical understanding. The idea of amalgamating these two broad ambits, the complexities of psychology and dynamism of Artificial Intelligence (Al), has gained momentum in recent years. The reluctance of a few psychologists in the past regarding inculcating expert systems into routine practice, because of employment-related insecurities or being swapped by a computer, led to the underutilization of the latent competence of using IT. Primarily, human behavior itself cannot be copied in totality by technology, and Al has a great deal to cover in this regards, but researchers are doing their best to deliver on these premises. Apart from this patient, resistance to this encroachment of technology should be critically investigated for gaining acceptance. This review focuses on how machine intelligence can enhance self-awareness through computer-implemented psychotherapeutic tools.
\end{abstract}

\section{Introduction}

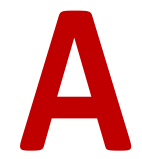

rtificial Intelligence (AI) has been introduced in multiple fields, including games, robots, law, stock trading, remote sensing devices, and scientific inventions, as well as diagnostic procedures [1]. Most of the advanced Al applications have greatly trickled into rou- tine applications so that they cannot be distinguished individually; once something is implemented as a general application, and it is of immense use, it cannot be specified as Al anymore. Al applications seem to have a ubiquitous infrastructural presence in any given industry. The late (1990s) and the beginning of the $\left(21^{\text {st }}\right)$ century were the start points of the intertwining Al technological elements into the larger frame of systems [1].

* Corresponding Author:

Mohammad Tahan, MSc.

Address: Young Researchers and Elite Club, Birjand Branch, Islamic Azad University, Birjand, Iran

Tel: +98 (912) 0252104

E-mail: t.mohammad2@gmail.com 
Interest in Al has primarily concentrated on autonomous systems that might replace people in the respective fields. Just as cognitive psychology may discover new avenues of investigation from algorithmic-instruction theory, algorithmic-instruction theory may have more practical implications as it assesses the complexity of human behavioral data. This human-centered focus is based on interdisciplinary synergistic research from cognitive neuroscience and psychology to theoretical computer science [2].

In this golden age of technology, with no end or limit in sight, the moral and ethical repercussions of Al have three sides to the argument. One opinion says that many of us are already stricken by poverty without work, and there is little or no reason to create mechanical laborers that can think independently. While the other says that society cannot progress or derive benefits from resources without the help of machines that can think for themselves at least partially. Moreover, the third group is least bothered about these issues at all, as is typical of human society [1].

Many psychologists think that the science of Al can integrate all of the phenomena generated by the human mind. The functionalist attitude witnesses the mind as a representational system and psychology as the offshoot of the various computational processes, whereby mental representations are built, organized, and deciphered. Although it is under debate as to how various psychological phenomena can be justified in computational terms; as to which Al concepts are applicable for computer modeling methodologies following psychologist's perspective is yet to be seen [3].

\section{Artificial Intelligence and Psychology}

A distinction is sometimes drawn between Al's engineering facet and its theoretical aspects. As an engineering discipline, the objective of $\mathrm{Al}$ is to devise and implement machines, which can perform operations by practical means. Accuracy, efficiency, flexibility, and reliability are the primary criteria of success for such systems and information about human performance seems to be neither necessary nor desirable [4].

The history of Al shows that program design and description has always relied on the elements of human psychology. Assumptions about cognitive processes have been drawn from the intuitions or introspective analyses of Al workers rather than empirical studies. Important programs, such as Samuel's checker-player were developed, applied, and tested without random- izing with any correlative studies of human performance [5]. Perhaps a part of the reason for Al's early disinterest in human studies was the indecisiveness for the full-fledged assimilation of psychology to underscore discrepancy in the constitution of intelligence. Gelernter's remarks affirm through the geometry-theorem proving program [6].

However, many Al workers have taken human studies into account; still, empirical psychology can be ignored in favor of intuition or introspection. Even those researchers, who explicitly acknowledge the significance of individual data, ignore psychological studies from their work. Winston (1979) suggested that the first of five steps in building an Al learning program is to examine or characterize some learning competence [7]. Winston's account of his program for replicating the application of simile in teacher-student interactions did not relate to any studies in educational psychology [8]. Instead, Winston rests on his intuition to describe the competence associated with such behavior. As ingenious as Winston's program is, it would be more compelling if it was derived from the empirical foundation of psychology [7].

Thus, calling attention for closer ties between Al and experimental psychology, it will confer an improvement in both aspects, i.e. in the principles of intelligent behavior as well as their respective computer applications. Raising psychological assumptions from the level of ad hoc intuitions to the level of systematic empirical observations will impart longitudinal improvement in terms of Al research quality and help to integrate it with related studies in other disciplines.

\section{A Precision of Task Definition and Description}

The past three decades have seen Al workers building (and reporting on) systems that utilize processes, to which they refer as reasoning, understanding, problemsolving, decision-making, planning, concept-formation, and so forth [9]. The terminological interpretation often alters from project to project, creating a patchwork of terminological customs. For instance, the term concept, as applied in the description of semantic networks, has no less than five significant interpretations. Such a loose application of psychological terms gives rise to three problems. Firstly, the comparison between different Al projects becomes extremely difficult. Secondly, the nature of task specifications, i.e. Al workers are fond of using psychological terms to associate their programs to aspects of human cognition explicitly and, thereby offering a pre-established theoretical framework [10]. 
If a researcher tries to conceptualize a program, he not only wants to improvise the implementation of a well-understood task but also intends to comprehend the first implementation. If the researcher characterizes his work as an understanding, many people may be misled, not to mention himself [11].

Program definition (or task environment) in this particular method gives rise to ambiguity because it tends to appeal to a psychological model when, in fact, it does not exist. This not only makes it incomprehensible the inherent lack of precision in the task specification but also gives rise to the third problem, namely the implicit reference to associated cognitive properties. When psychological terms, beliefs, or understandings are casually employed to define or describe an Al program, it is easy to succumb to the temptation to assign a cluster of related cognitive properties to it. This is almost unavoidable since the pre-theoretic or lay of psychological terms occurs only regarding the human (or animal) cognition. It is hard to make sense of many of these terms without presupposing an integrated background of cognitive phenomena, but when we consider them in an $\mathrm{Al}$ context, such a background is missing [12].

\section{The Use of Human Data as a Methodological Heuristic}

The distinction between $\mathrm{Al}$ as an engineering discipline and $\mathrm{Al}$ as a philosophical inquiry is conducted in the light of the methods employed, rather than in the perspective of the tasks employed. For instance, one could take an engineering strategy to visual pattern recognition, game-playing, or natural language understanding, just as one could take a theoretical approach to automated programming, tree-searching, or even robotics. The task specifications or programming techniques are in no way responsible for the unlikeness of the two approaches to $\mathrm{Al}$; on the contrary, it depends on the employed method and the researcher's objective [4]. However, commonalities between these two domains cannot be negated. The cognitive approach is a crossing point in psychology. Within this domain, applicability involves a simulationbased environment learning, computer-based emotion recognition, intra-group social interaction simulations, cognitive-behavioral therapies, computer-based psychiatric therapy, electronic inquiries as well as automatic output generation, and so on.

The findings of National Institute for Health and Care Excellence (NICE) (2008) regarding the IT applicability in cognitive behavior therapy has yielded promising outcomes. Novel spheres of research like cyberpsychology have emerged, reflecting the acceptance of IT in the field of psychology by the research fraternity. Psychotherapy inpatient treatment has adopted two distinct levels of IT applications [13]. Firstly, the application is engaged in refining the efficacy, execution, and performance of the practicing therapist. Secondly, it is associated with complex systems that resolve issues the patient and the therapist might get enmeshed in during treatment. Once a few reservations are clarified, the expert systems are expected to solve low and medium complexity issues [14].

Today, the youth are more techno-savvy and more comfortable with different types of information technologies. Data retrieval in corporate offices is easier now at the click of a button. This easy accessibility to a broad purview of research data of thoughts, feelings, and individual/group behavior by a psychologist is lacking and needs to be addressed [15]. Therefore, newer tools are being embraced by psychologists to collate social psychology data as well as related fields of psychology. Therefore, to bridge the gap, a coordinated intelligent interface comprising of expert and information retrieval system is the need of the hour with a requirement of an additional interface design agent [15].

\section{Information Technology and Psychology}

The computer is gradually entering into psychology and psychiatry research or treatment. Not all experts consider this development as an utterly positive step [16]. These opinions are cross-examined with the intent to solve it. Direct patient-therapist interaction is the mainstay of psychotherapy; this arrangement is, however, being substituted by IT tools because of the permeation of information society in our routine life. As a result, progressive human-computer interaction is only the beginning of things to come, possibly even reduce cash flows of this class of experts. Adaptation to this scenario in the next two decades to attain a new equilibrium point is expected. Still, the computer has a long way to go to match the flexibility and dynamism of a human mind; quality might be the first casualty in the human-computer interaction. Readapting the treatment schemas could attempt to harness the benefits offered by the novel computerized system and suppress its undesired effects. The quality of treatment is the primary cause for concern in computer-assisted or computer-replaced psychotherapy, while longitudinally organizational resistance seems to pose as a minor problem associated with it [15].

The patients' resistance cannot be underestimated, but as time passes, information technology will be integrated 
into people's life from inception, and human-computer interaction will only become secondary. This type of rejection among the clinician community was previously witnessed with the testing of Micyn (the very first automated diagnostic system) [17]. Unfortunately, to steer clear of possible misdiagnosis, Micyn use was not implemented $[18,19]$. The diagnostic accuracy of 99\% is achievable by implementing an expert system, but in the similar circumstances, practitioners might need a comprehensive record, thereby indicating their requirement only as an aid in the process rather than an option or replacement.

Nevertheless, a new field came into existence in social science, i.e. cyberpsychology or the psychology of cyberspace. Studying people's reaction and their cyberspace behavior is another evolving concept created by computers and online networks [20]. Cyberpsychology research is directed to solve two main aspects, i.e. enhanced applications of IT to counter various psychological issues and the consequences to the use and interaction of various tools of the cyberspace and associated psychological and psychiatric issues among the user.

With the merger of the information society in each aspect of routine life, this new concept was a natural progression with the ever-increasing and diverse studies related to the subject of applicability of IT by psychologists [17]. The justification of huge capital investments in the implementation of these complex systems and the dilemma with regards to the expected delivery of these systems in terms of the actual needs of the psychology expert are inevitable in terms of complexity and purpose of the typical IT applications in real-world settings. The predicament of adaptability of the user to the system complexity is another problem faced in the given context. Tests on system efficiency by employing a minimal prototype can perhaps clear the first doubt while the user's rejection of an issue can be elucidated by implementing specific features for the Human-Computer Interface $(\mathrm{HCl})$. Although only on a gadget level, a good information system based on an expert system is needed, without which even the electronic documentation, known as Help, cannot resolve this issue.

A more interactive approach is expected from the application of Intelligent Tutoring Systems (ITS). An expert system-dependent ITS with the touch of a finger from the massive combination between authoring event and psychology; to boost the competence in handling, the customized help offered to the teacher to devise new materials and its appropriate application in ITS is needed [17].
Psychology-based computer applications are mainly referred to as psychotherapy. The computer at this juncture is far more relevant than human in a similar space. The roots of $\mathrm{Al}$ in cognitivism have made the psychiatrists employ the computer only as an ancillary support system during treatment. A battery of broadbased applications can be classified as follows: dedicated websites for self-help; computer-administered therapy; web-based applications for identifying and evaluating via the Internet, advocacy, adjunctive palmtop computer therapy; consultation via the Internet; Virtual Reality (VR) therapy; interactive voice messaging systems; biofeedback via ambulatory physiological monitoring; and virtual support group sites. Essential advantages by the use of IT in psychotherapy include more time gained by the patient for supervised treatment, shorter interaction with the practitioner, cost-effective treatment, assisting in making treatment decisions. The idea of employing the computer to support the expert is not novel, for instance, in the application of lengthier interviews [2].

The shortcomings of the application of computer in psychotherapy are primarily related to ethical issues; the patient-therapist bonding is where a lacuna lies and the discretion of leaving an entire human being into this form of relationship is interrogated [21]. Then, considering the availability and easy access of the software to the patient at free will is akin to the case of drugs that can be used only under continuous medical supervision because of the predisposing risk factors. Computer models tend to oversimplify things, and the odds of reducing the adaptability and ability of the human expert are much higher. Consequently, with passing time, there is a higher likelihood that the expert will not be able to comprehend the patient's issues without the aid of the computer. The benefits of the computer-based application at any level in psychology cannot be ignored, but appropriate care is given to the rising expectations from this particular approach. Given the gravity and complexity of psychological issues, limitations associated with the system cannot be neglected; for instance, problems like nightmares, compulsive gambling, tics, and enuresis have not been resolved till now.

It means that despite the utility of these systems, continuous updating is required; however, substitution by the specialist is not feasible at the moment. The hypnotherapy may be carried out classically, but excellent outcomes are also gotten by the application of electronic techniques either partially or totally. Considering the computer have fully automated audio/video flow, thus reflecting the higher IT involvement. Table 1 represents 
Table 1. Computer-based hypnotherapy application

\begin{tabular}{ccc}
\hline Psychological Issue & Techniques Recommended & Applied Methods \\
\hline Stress & Self-hypnosis & Interactive web applications \\
Anxiety & Hypnotherapy & Interactive web applications \\
Depression & Relaxation therapy & Stand-alone applications \\
Phobias on various forms & Meditation & Multimedia support \\
Cognitive issues (e.g. positive thinking) & Stress management & Mini mixing desks
\end{tabular}

the techniques recommended for psychological issues and methods employed in conjunction with a computer [22]. To help the psychotherapist, health care services have allowed access to VR; the specialists have approved the VR role and think that it might further refine and evolve the field of clinical psychology.

\section{Al Applications in Psychology}

The outcomes of the panic and phobia disorder treatment were not on a par with the expectations from the computer-based application, even though they were much economical.

Children's age-based educational games have become regular and hold many potentials, which the psychiatrists must explore. The concept of employing games in education with increasing the level of complexity is being applied. These games are, more often than not, based on complex expert systems or a variant of advanced Al. The psychologists have to integrate these strategies. Studies related to the applicability of 3D games as a resolute therapy instrument have been conducted [23]. The first outcome seems to be promising; nonetheless; it is an uphill task to find a general treatment solution. Therefore, the psychiatrist-guided supervision of behavioral rule modification of the therapeutic games is required from time to time.

The superiority of Al dynamics against psychology can be attributed to its strong mathematical backbone and important industrial applications. The (1980s) saw the rise in the use of an expert system as a market asset after that of production systems [24]. Expert systems and psychology cannot be separated. Over time, IT experts have realized that there is a need for competent rule extraction from people to devise new techniques. Here, the Repertory Grid elicitation (RepGrid), which can yield data for analysis, quantitative and qualitative were identified and integrated into the historical understanding.
From the psychological perspective, the expert systems can be implemented coupled with personal construct psychology. It should not be forgotten that these psychologist approaches do not come cheap. So, a middle path has to be taken for the expert system with generalized rules about human, and the thinking pattern is made; later, a form of self-acquiring guidelines from direct interaction with the patient will be implemented.

The expert systems are complex applications that select a set of guidelines based on the human expert experience in given circumstances. Apprehensions regarding its implementation are usually applied to the dimension of the set of guidelines and eventually to the conferring clarity of this set. From the computing dimensions, high-performance computing such as Grid or Cloud can achieve great heights. However, human thoughts are a very complex subject to know, especially it cannot be put down in words, and the absence of a communication channel may further limit the knowledge transfer. From a theoretical point of view, the applicability of these systems is vast, because the basic idea behind the development of Al could imitate human thinking; but, to achieve this feat, we have a long way to go. Various branches of Al are trying to copy some parts of life behavior, from genetic algorithms and neural networks to artificial life, fuzzy, and game theory.

Any expert system must comprise three key components; the knowledge base, the inference engine, and the interface. The universal translator seems to be the first application in the pipeline. The level of knowledge offers to resolve a vast and varied database like Google and complemented by an equally formidable expert system. The expert systems application in speech therapy can further enhance its use. Researchers hope that in the light of this improved fuzzy, an expert system can also be applied in providing at-home treatment of the patient [25]. 
Different Al techniques are used in general psychiatry. For instance, even low-quality input data are enough in the correct diagnosis of dyslexia by merging the applicability of fuzzy and genetic algorithms. The passive voice itself can be used as an auxiliary source of information in creating a good anamnesis. Voice pathology is also being used in finding important psychological cues; the outcomes of Massachusetts Eye and Ear Infirmary (MEEI) Voice Disorders Database is one such example [26]. These outcomes cannot be separately treated as multiple reasons can be responsible for the change in a patient's voice. Its use can give valuable information about the patient if used concomitantly with other measurements.

\section{Social Information Retrieval System}

Social sciences and psychology experts have to stay updated with the current cyberspace scenarios. The data collection of people or communities is central, and newer ways must be devised; today, information retrieval from the Internet is also possible. Social life is either partially or fully being virtualized so that personal details can easily be accessed online. These sets of information can be distinguished into explicit data or implicit data; the former is required by the social network so that the user knows about the content made public and is made aware about the implications of making them partially or fully public. While the former data involve the information given by way of interaction with all those acquainted via the social network platform.

Inability to distinguish between the virtual world and direct contact with the group members is the biggest threat to the users because they may not be discerning about the type and the confidentiality of the information made available. The virtual space has the information, thereby an interface with the social network must be created. Accessing these personal details of the user without his/her consent is not possible without obtaining explicit permission. Two segments make the proposed system: the first one is the $\mathrm{HCl}$-based interface, using intelligent agents, and the second one is the information retrieval system. The convenience of the use of a computer is possible by using expert systems and $\mathrm{HCl}$ techniques. Too much investment is not required to gain direct or indirect benefits in case of higher emotional intelligence in some individuals. Therefore, experts emphasize a computer capable of emulating these kinds of abilities.

\section{An Information Retrieval System}

The Information Retrieval System (IRS) system comprises the data gathering, indexing, searching, and presentation. The data are gathered by retrieving information from the Internet, or local networks as per the users set rules. Occasionally, it is applied as the solution of search by using independent units that will transfer the information to the central database. This case may require a data normalization process and some pre-indexing algorithms. In indexing, people look at the immediately searchable database. Although varied indexing approaches exist, the relevance of each depends mostly on the data size. Therefore, a traditional DataBase Management System (DBMS) is used to store data. In searching, a dedicated set of Al-based operations are used for each implicit DBMS users. In the presentation, the graphical user interface employed in graphical data representation is made; clustering methods are also used. Cyberpsychology is a related research field still in infancy with unlimited potential because of the high speed of technological development [23].

\section{Psychotherapy and Al Alignment}

The process of familiarizing with the patient involves the designing of mental models based on the small bits of available information. Al prioritizes the modeling of knowledge since understanding human behavior is integral for the machine to imitate. Knowledge Geometry is the computer-designed model of representation based on concepts (intuition) and vice versa (reification). The professional patient assesses the patient behavior patterns and map them on the clear conditions and subjects them to brief psychotherapy. The theoretical concepts of psychotherapy are applied in cases, where conflicts arise between the prepared psychotherapy maps and the individual in question. The reification operation is a process of inference, where analogies and isomorphism database are used as a resource. By analyzing each process and family pattern, it can be crossexamined, and novel methods can be devised for the system. The mechanisms of feedback and maintenance do not allow the system to obtain newer experiences and learning, thereby stunting its development or solving the conflict in question. This is referred to as casebased reasoning and is done by first-order logic. While the psychotherapist tries to simplify the patient's symptom and associate it to the broader interactional system and link it to the global scenario, it reflects the intuition operation of Knowledge Geometry. 
This particular phenomenon is later applied as ancillary support for understanding a broader pattern in this case; Al calls a process akin to machine learning. The systemic-linking method is a step forward in the application of computational intelligence as an auxiliary tool in evaluating the behavioral pattern of couples, families, and individuals. Among the numerous strategies of Al, first-order logic, automatic theorem prover, and fuzzy logic can be used to match the associated psychotherapy interventions linked to the characterization of essential areas, genogram creation, self-determination assessment, cognitive interaction pattern testing, improved self-awareness, and modifications [24, 25]. Despite these developments, the opinion that Al investigations have not yet evolved as a universal method for sophisticated formulation of problems needs not to be overlooked [26].

\section{Discussion}

As with any emerging technology, caution needs to be used judiciously to overcome optimistic biases and, primarily to serve the best interests of the people, for whom the technology is designed to help. Not to mention that Al is here to stay, further emphasizing that the organization must stay attuned to the economic and social changes of the society; otherwise, it will fade away.

Nevertheless, psychology will always be a constant source of help to individuals struggling with insecurities and societal adjustments. Moreover, the advanced technological development has not in any way altered the mutual stimulus established by patient-therapist interaction. Technological advancements influence psychologists in many ways; however, the effect of the advancement in technology on psychology is associated with its own set of positive and negative aspects, for which the clinicians, therapists, counselors, and researchers must be well-equipped to handle.

\section{Ethical Considerations}

\section{Compliance with ethical guidelines}

All ethical principles were considered in this article. The participants were informed about the purpose of the research and its implementation stages; they were also assured about the confidentiality of their information; Moreover, They were allowed to leave the study whenever they wish, and if desired, the results of the research would be available to them.
Funding

This research did not receive any specific grant from funding agencies in the public, commercial, or not-forprofit sectors.

\section{Authors' contributions}

All authors contributed in preparing this article.

\section{Conflict of interest}

The author declare that the research was conducted in the absence of any commercial or financial relationships that could be construed as a potential conflict of interest.

\section{References}

[1] Shukla SS, Vijay J. Applicability of artificial intelligence in different fields of life. International Journal of Scientific Engineering and Research. 2013; 1(1):28-35

[2] Mozer MC, Wiseheartd M, Novikoffc TP. Artificial intelligence to support human instruction. Proceedings of the National Academy of Sciences. 2019; 116(10):3953-5 [DOI:10.1073/pnas.1900370116] [PMID][PMCID]

[3] Wang Z, Xie L, Lu T. Research progress of artificial psychology and artificial emotion in China. CAAI Transactions on Intelligence Technology. 2016; 1(4):355-65. [DOI:10.1016/j.trit.2016.11.003]

[4] Tran BX, Vu GT, Ha GH, Vuong QH, Ho MT, Vuong TT, et al. Global evolution of research in artificial intelligence in health and medicine: A bibliometric study. Journal of Clinical Medicine. 2019; 8(3):360. [DOI:10.3390/jcm8030360] [PMID] [PMCID]

[5] Lee Y, Ragguett RM, Mansur RB, Boutilier JJ, Rosenblat JD, Trevizol A, et al. Applications of machine learning algorithms to predict therapeutic outcomes in depression: A meta-analysis and systematic review. The Journal of Affective Disorders. 2018; 241:519-32 [DOI:10.1016/j.jad.2018.08.073] [PMID]

[6] Gelernter H, Hansen JR, Loveland DW. Empirical explorations of the geometry-proving machine. In: Feigenbaum E and Feldman J, editors. Computers and Thought. New York: McGraw-Hill; 1963.

[7] Winston PH. Learning by creating and justifying transfer frames. In Winston PH and Brown RH, editors. Artificial Intelligence: An MIT Perspective. Cambridge, Massachusetts: MIT Press; 1979.

[8] Klausmeier HJ, Ghatala ES, Frayer DA. Conceptual learning and development: A cognitive view. New York: Academic Press; 1974.

[9] Luxton D. Artificial intelligence in psychological practice: Current and future applications and implications. Professional Psychology: Research and Practice. 2014; 45(5):332-9. [DOI:10.1037/a0034559]

[10] Hendrix GG, Lewis WH. Transportable natural language interfaces to databases. Stroudsburg: ACL 81 proceedings of the $19^{\text {th }}$ annual meeting on Association for Computational Linguistics; 1981:15965. [DOI:10.3115/981923.981965] 
[11] McDermott, D. Methodological polemic, presentation to the artificial intelligence society of New England. New Haven: Yale University; 1980.

[12] Dasilva G, Dwiggins D. Toward a prolog grammar. SIGART Newsletter. 1980; 73:20-5.

[13] Howell SR, Muller R. Computers in psychotherapy: A New prescription. Ontario: McMaster University Hamilton; 2000.

[14] Marks M, Cavanagh K, Gega LI. Computer-aided psychotherapy: Revolution or bubble? British Journal Of Psychiatry. 2007; 191:4713. [DOI:10.1192/bjp.bp.107.041152] [PMID]

[15] Zaharia MH. Al Applications in psychology, expert systems for human, materials and automation [Internet]. 2011 [Updated 2011 October 14]. [DOI: 10.5772/16620] Available from: https://www. intechopen.com/books/expert-systems-for-human-materials-andautomation/ai-applications-in-psychology

[16] Suller J. Psychology of the digital age: Humans become electric $1^{\text {st }}$ Edition. New Jersey: Rider University; 2015. [DOl:10.1017/ CB09781316424070]

[17] Rialle V, Stip E, O'Connor K. Computer-mediated psychotherapy ethical issues and difficulties in implementation. Humane Medicine. 1994; 10(3):185-92.

[18] Frost B. Computer and technology enhanced hypnotherapy and psychotherapy. A review of current and emerging technologies: 2008. [Internet]. 2008 [Updated 4 February] Available from: www.neuroinnovations.com/ctep/technology_and_computer_enhanced_psychotherapy.pdf

[19] Coyle D, Matthews M, Sharry J, Nisbet A, Doherty G. Personal investigator: A therapeutic 3D game for adolescent psychotherapy. Journal of Interactive Technology \& Smart Education. 2005; 2(2): 73-88. [DOI:10.1108/17415650580000034]

[20] Shaw ML, Gaines BR. Expertise and expert systems: Emulating psychological processes. In: Fransella F. editor. The Essential Practitioner's Handbook of Personal Construct Psychology. New York: John Wiley \& Sons Inc; 2005.

[21] Schipor OA, Pentiuc St, Gh, Schipor DM. Fuzzy rules base for computer based Speech therapy. Paper presented at: Proceedings of $9^{\text {th }}$ International Conference on Development and Application Systems. May 24-26 2018; Suceava, Romania.

[22] Saenz-Lechon N, Godino-Llorente Jl, Osma-Ruiz V, Gomez-Vilda P. Methodological issues in the development of automatic systems for voice pathology detection. Biomedical Signal Processing, and Control. 2006; 1(2):120-8. [DOI:10.1016/j.bspc.2006.06.003]

[23] Kowalski G. Information retrieval architecture and algorithms. Boston: Springer; 2011. [DOI:10.1007/978-1-4419-7716-8_8]

[24] D'Alfonso S, Echarri SO, Simon R, Wadley G, Lederman R, Christopher $\mathrm{M}$, et al. Artificial intelligence-assisted online social therapy for youth mental health. Frontiers in Psychology. 2017; 8:796 [DOI:10.3389/fpsyg.2017.00796] [PMID] [PMCID]

[25] de Mello FL, de Souza SA. Psychotherapy and artificial intelligence: A proposal for alignment. Frontiers in Psychology. 2019; 10:263. [DOI:10.3389/fpsyg.2019.00263] [PMID] [PMCID]

[26] Dwyer DB, Falkai P, Koutsouleris N. Machine learning approaches for cinical psychology and psychiatry. Annual Review of Clinical Psychology. 2018; 14:91-118. [DOI:10.1146/annurevclinpsy-032816-045037] [PMID] 